\title{
Derangement of body representation in complex regional pain syndrome: report of a case treated with mirror and prisms
}

\author{
Janet H. Bultitude $\cdot$ Robert D. Rafal
}

Received: 16 April 2009 / Accepted: 17 November 2009 / Published online: 6 December 2009

(C) Springer-Verlag 2009

\begin{abstract}
Perhaps the most intriguing disorders of body representation are those that are not due to primary disease of brain tissue. Strange and sometimes painful phantom limb sensations can result from loss of afference to the brain; and Complex Regional Pain Syndrome (CRPS) - the subject of the current report - can follow limb trauma without pathology of either the central or peripheral nervous system. This enigmatic and vexing condition follows relatively minor trauma, and can result in enduring misery and a useless limb. It manifests as severe pain, autonomic dysfunction, motor disability and 'neglect-like' symptoms with distorted body representation. For this special issue on body representation we describe the case of a patient suffering from CRPS, including symptoms suggesting a distorted representation of the affected limb. We report contrasting effects of mirror box therapy, as well as a new treatmentprism adaptation therapy-that provided sustained pain relief and reduced disability. The benefits were contingent upon adapting with the affected limb. Other novel observations suggest that: (1) pain may be a consequence, not the cause, of a disturbance of body representation that gives rise to the syndrome; (2) immobilisation, not pain, may precipitate this reorganisation of somatomotor circuits in susceptible individuals; and (3) limitation of voluntary movement is neither due to pain nor to weakness but, rather, to derangement of body representation which renders certain postures from the repertoire of hand movements inaccessible.
\end{abstract}

J. H. Bultitude $(\bowtie) \cdot$ R. D. Rafal

Wolfson Centre for Clinical and Cognitive Neuroscience,

School of Psychology, Bangor University,

Brigantia Building Penrallt Road, Bangor,

Gwynedd LL57 2AS, UK

e-mail: j.bultitude@bangor.ac.uk
Keywords Complex regional pain syndrome . Mirror therapy $\cdot$ Prism adaptation $\cdot$ Body representation

\section{Introduction}

S. Weir Mitchell first described a syndrome of chronic allodynic pain (that he called causalgia due to the burning quality of the pain) in soldiers of the American Civil War with injuries to peripheral nerves (Mitchell et al. 2007). Sudeck (1900) described the same symptoms following limb fracture without nerve damage. This became known as Sudeck's Atrophy, and subsequently, with the finding that pain relief could be provided by surgical sympathectomy, as Reflex Sympathetic Dystrophy (RSD; Evans 1946). For over a century these and several other terms were used to describe similar sets of symptoms (see Lam et al. 1996), until in 1994 the International Association for the Study of Pain (IASP) established a new title, Complex Regional Pain Syndrome (CRPS), distinguishing between cases that follow nerve damage (type II, formerly causalgia) or in which no obvious nerve damage has occurred (type I, formerly RSD; Stanton-Hicks et al. 1995).

CRPS has been observed after sprains, surgery, frostbite and other soft tissue injuries (de Mos et al. 2007; Choi et al. 2008), and has also been reported in cases where no precipitating injury has occurred (Veldman et al. 1993). It can emerge weeks after the limb has healed. The pain is disproportionate to the severity of trauma, and is associated with swelling and trophic changes of the skin and nails, neurogenic inflammation, osteoporosis as well as autonomic dysregulation: the limb becomes sweaty and is warm due to decreased sympathetic tone; in later stages it becomes cold due to denervation or hypersensitivity to noradrenaline. Symptoms can resolve within a few months of onset, 
although the proportion of patients for whom this can be expected is uncertain, as clinical awareness of CPRS is still poor (Manning 2000). Patients experience symptoms for an average of 2 years before being referred to a pain centre, by which time they will have seen five doctors (Choi et al. 2008). Nonetheless, it has been estimated that $50 \%$ of cases develop into a chronic syndrome of pain and disability (Schasfoort et al. 2004).

The consensus diagnostic criteria established by the IASP include a combination of (1) the presence of an initiating noxious event or cause of immobilisation, (2) continuing pain, allodynia or hyperalgesia that is disproportionate to any inciting event, (3) evidence at some time of oedema, changes in skin blood flow or abnormal sudomotor activity in the region of the pain, and (4) absence of a condition that would otherwise account for the degree of pain and dysfunction. The presence of a known nerve injury leads to the diagnosis of CRPS type II, with CRPS type I diagnosed where no nerve injury has occurred (Stanton-Hicks et al. 1995).

Without aggressive physiotherapy and adequate pain control, the limb can become paralysed, cold, and sweaty, and can assume dystonic postures, rendering it permanently useless and an enduring misery (Doury 1988; Gordon 1996; Berger 1999). Corticosteroids, amitriptylene and gabapentin provide some relief in some patients, and approximately $85 \%$ of patients receive some symptomatic relief from sympathetic blockade if instituted early, indicating that their pain is at least partially maintained by sympathetic mechanisms (AbuRahma et al. 1994). The degree of this improvement can vary dramatically between individuals, and are often transient (Wasner et al. 2003), and other patients do not respond to sympathetic blockade at all (i.e., sympathetically independent pain; Torebjork et al. 1995).

Identifying the pathophysiology of the disorder has been elusive, to the point where historically cases have been attributed to malingering or catastrophic psychological reactions to the trauma (for a review, see Bruehl and Carlson 1992). However, CRPS is now recognised as a biological disorder, with the discovery of disease mechanisms such as local nerve degeneration (van der Laan et al. 1998; Oaklander 2008) and elevated levels of inflammatory mediators in cerebrospinal fluid (Alexander et al. 2005) and in the affected limb (Huygen et al. 2002).

Some symptoms of CRPS suggest that the neural dysfunction is not limited to the peripheral and autonomic nervous system but that there is also a reorganisation of cortical function. Some of these manifestations include symptoms that are referred to as 'neglect-like' because they resemble those seen in patients with hemispatial neglect. Half of interviewed patients reported feeling that the afflicted limb seemed foreign or did not seem to belong to them; and half had difficulty identifying which finger was touched (Forderreuther et al. 2004). Motor neglect-a dearth of spontaneous movement of the limb resulting in disuse that aggravates the problem-is often present. Patients report difficulty moving their fingers unless they look at them. Paradoxically, when asked to position a visual target straight ahead of the body midline, patients erred towards the affected side, suggesting a pathologically altered perception of body midline in which the affected side of space is over-represented (Sumitani et al. 2007).

Some symptoms suggest a distortion of body image (Lewis et al. 2007). When patients chose the 'accurate' picture from a selection of photographs of their forearms and hands in which the width of the affected limb had been compressed or expanded, their choices reflected an overestimation of limb size (Moseley 2005). Range of motion can be increased by making movements with both hands while viewing their unaffected hand in a mirror (McCabe et al. 2003b) thus producing the illusion of a normally functioning hand. Pain can be precipitated by viewing an object approaching the limb, and the pain increases as the object gets closer (McCabe and Blake 2008). Movement-induced pain is aggravated by viewing the limb through lenses that make the limb look bigger, and reduced by viewing it through a minimising lens (Moseley et al. 2008).

These observations suggest that the brain has been pathologically reorganised such that there is an extension of the neural representation of peripersonal space (the space within grasp). Touch to the affected limb can induce referred sensation in body areas with neighbouring cortical representations (McCabe et al. 2003a). Functional neuroimaging studies have shown that the representation of the affected limb on motor cortex is enlarged (Pleger et al. 2005), while the representation on primary sensory cortex is reduced (Maihofner et al. 2003). This reorganisation appears to be both reversible and directly related to pain symptoms: the degree of pain reduction following rehabilitation correlated with normalisation of the extent of primary sensory cortex (Maihofner et al. 2004).

Recently, two therapeutic interventions for CRPS have been introduced to relieve pain and other symptoms: mirror box therapy and prism adaptation. Both are based on manipulating sensory-motor information concerning the affected limb. Mirror box therapy was initially introduced to treat phantom limb pain (Ramachandran et al. 1995). For patients with CRPS, the treatment can enhance range of motion, and may also reduce pain in some patients (McCabe et al. 2003b; Tichelaar et al. 2007). The patient executes synchronous bimanual movements while viewing the reflection of the unaffected hand in a mirror placed in the sagittal midline. The patient 'sees' their affected limb executing the full range of movement and, indeed, the affected 
hand does execute deft movements with the injured hand in synchrony with the unaffected hand.

The prevalence of 'neglect-like' symptoms in CRPS, and their observation that CRPS patients exhibited deviations in perceived body midline, led Sumitani et al. (2007) to undertake a trial of prism adaptation therapy in five patients.

When patients with neglect undergo adaptation to rightward-shifting prisms, resulting in a leftward visuomotor after-effect, improvements are observed in neglect symptoms (Rossetti et al. 1998). This improvement has been well replicated for many aspects of neglect, including perception of body midline (Pisella et al. 2002; Sarri et al. 2008), tactile sensation (Maravita et al. 2003), haptic exploration (McIntosh et al. 2002), and finger position sense (Dijkerman et al. 2004).

Sumitani et al. (2007) demonstrated that 2 weeks of daily adaptation to $20^{\circ}$ prismatic shifts that induced an after-effect towards the affected limb relieved the pain and autonomic dysfunction in five patients with CRPS and reduced their pathological perceptions of body midline. In a further longitudinal study of a single patient they found that adaptation to neutral or $5^{\circ}$ refracting lenses did not produce any effects, and adaptation towards the affected side exacerbated pain.

Here we report observations on the benefits of both mirror therapy and prism adaptation in a woman with CRPS type I over 15 weeks in which she underwent periods of daily prism adaptation, and adaptation-free periods. We report several novel observations, including a dissociation between pain and motor disability, that may shed light on the derangement of body representation in CRPS, and the role of this derangement in the pathogenesis of CRPS symptoms.

\section{Case report}

$\mathrm{SM}$ is a 53-year-old right handed woman who we first examined 5 months after she sustained fractures of the right hand. In May 2008 the hammock she was lying in collapsed. She was concussed when a metal hook struck her behind the right ear. She heard the crack of the wood hammock breaking but does not remember the fall to the ground or the blow to the head. She was dazed and unable to communicate for about half an hour, and was then able to phone for help. A piece of wood struck her right hand, resulting in spiral fractures of the third and fourth metacarpals. The hand was splinted for 3 weeks, when the splint was discarded because the orthopaedic surgeon observed that 'she is stiffening up considerably'. A week later her physiotherapist noted reduced range of motion, swelling of the hand and tenderness to light touch (allodynia). Subsequent X-ray confirmed complete healing of the fracture in the fourth and near complete healing of the third metacarpal.

She had been fit prior to this injury. However, at the age of seventeen, SM sustained a traumatic amputation of the entire index finger and half of the middle finger of her left (unaffected) hand. This had been followed by the occasional sensation of phantom pain in the missing index finger, which decreased in frequency with time and which SM described as having been only a mild annoyance. At the time of the present study SM had not experienced any phantom digit pain for many years.

During the time that she was wearing the splint, she noted that there were times when she did not 'know where my hand was'. SM reported being unable to sense what her hand was doing, and had difficulty visualising its position when she was not directly looking at it. The pain from her injury was much improved by the time the splint was removed. However, shortly thereafter, she began to experience a different kind of pain in the hand. Initially there were intermittent paroxysms of sharp burning pain in the palm, but eventually the pain became continuous and affected her fingers, hand and forearm. Three months after her injury, her physiotherapist prescribed daily mirror therapy (see below) upon suspicion of CPRS, a diagnosis that was confirmed by the Hand and Orthopaedic Surgeon.

When we first examined her, she had been experiencing months of constant pain in her right forearm and hand. This she described as being dull and 'like a toothache you can just about bear most of the time', and rated as four on a subjective numerical rating scale (NRS; with 0 indicating no pain at all, and 10 indicating pain as bad as it could be). Several times a day she would experience a sharp increase in pain $(\mathrm{NRS}=7)$ that was burning in quality, with the focal point varying from day to day. This could occur spontaneously; however, opening and closing her hand also caused a sharp pain. Amitriptylene had provided no relief and she required narcotic analgesic mediation at least once a day. Mirror therapy had also failed to provide pain relief.

Medical examination revealed swelling of the right hand and wrist. On palpation, the right hand was warmer than the left and the palm was sweaty. No discolouration or trophic changes were observed, although SM reported that at times her right hand appeared 'almost blue' compared to the left. Movements were slow and clumsy, with particular impairment of fine finger movements, and she was unable to fully open or close the hand. The restriction in range of motion was greater for hand closure than opening. Range of movement was also better when SM was using objects than when performing actions with an empty hand: she could achieve more closure grasp when using objects than when asked to close her fist on verbal command. Light touch sensation was intact and there was no tactile extinction. Atypically 
for CRPS, allodynia was not conspicuous: light touch did not produce much discomfort. There was, however, hyperalgesia: mild pin prick discomfort was experienced as painful. Position sense and two-point discrimination were less acute in the injured right hand compared to the left hand. An ultrasound confirmed mild degenerative changes and subcutaneous swelling. In summary, SM suffered from pain disproportionate to her injury, and examination revealed pain, hyperalgesia, swelling, temperature changes and other symptoms that were disproportionate to SM's original injury and were consistent with the IASP diagnostic criteria for CRPS (Stanton-Hicks et al. 1995). SM also demonstrated several motor and sensory abnormalities that have frequently been associated with the disorder.

\section{Mirror therapy observations}

Full range of movement was restored when SM made synchronous opening and closing movements of her hands while viewing the reflected image of her unaffected left hand. This was unaccompanied by the pain that she usually experienced with movement. She was able to fully close the right hand while using the mirror. However, this ability was lost as soon as the mirror was removed. Indeed, if she closed her right hand while using the mirror, she was unable to open it after the mirror was removed-even though this would only have required that she be able to relax her hand. Mirror therapy did not facilitate movement of the affected hand when attempted while viewing the reflection of the examiner's moving hand in the mirror, nor if she attempted bimanual synchronous movements without the mirror. However, it was effective when viewing the reflection of the examiner's moving hand, if she simultaneously executed the movements with her unaffected hand while it was out of view under the table. Thus, the efficacy of mirror therapy was not dependent upon the hand viewed in the mirror being in the homologous position to the injured hand, or looking like her own injured hand. Indeed, mirror therapy was effective despite the obvious visual differences between the affected and unaffected hand due to the missing digits on the left (unaffected) hand.
Rather, efficacy was dependent upon the execution of bimanual, synchronous movements while simultaneously perceiving the visual illusion of normal movement in the injured hand.

Mirror therapy also provided momentary relief of pain and stiffness, which unfortunately resumed almost immediately after the mirror was removed. However, SM did find it helpful to perform mirror therapy 2-3 times a day for approximately two minutes per session to relieve the stiffness in her hand.

Prism adaptation

\section{Design and procedure}

CRPS symptoms were formally assessed in nine sessions spanning 15 weeks, during which the following conditions were applied: Treatment (3 weeks), washout (13 days), treatment using the unaffected hand ('left-hand treatment', 1 week), treatment ( 9 weeks; see flow chart in Fig. 1). This sequence was not based on a pre-determined research protocol. The trial of prism adaptation was offered to the patient based on clinical consideration and solely for the purpose of potentially alleviating her misery. Decisions on how to apply the therapy were made based on response to therapy and clinical judgment. (The one exception to this was a brief trial of adaptation with the unaffected hand.)

For each clinical assessment (CA1-CA9), oedema and discolouration were evaluated by visual comparison to the unaffected hand. Range of movement (ROM) was also assessed by direct visual comparison, for which SM was asked to open and close both hands several times. To assess temperature, the examiner simultaneously touched the dorsal surfaces of the fingers of each hand to the upturned palm of SM's left and right hands. In addition to the formal assessments SM recorded daily ratings of pain and ROM in her right hand (Fig. 2). These were recorded as average ratings for the previous $24 \mathrm{~h}$ on scales from 0 ('no pain at all'/'no movement at all') to 10 ('pain as bad as it could be'/'completely normal movement'). She continued normal use of mirror therapy as described above, and medical pain relief as required.

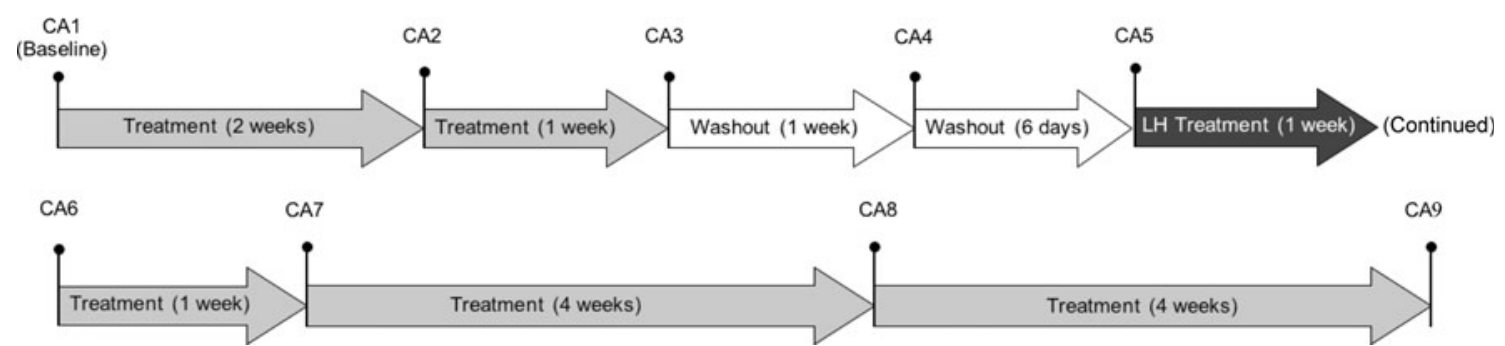

Fig. 1 Treatment schedule and timing of clinical assessments (CA1CA9). In 1-week blocks SM underwent daily sessions of adaptation to leftward-shifting prisms with her affected hand ('treatment', light

grey), with her unaffected hand ('left-hand treatment', dark grey), or no prism adaptation ('washout', white, 6 days only in the second block) 


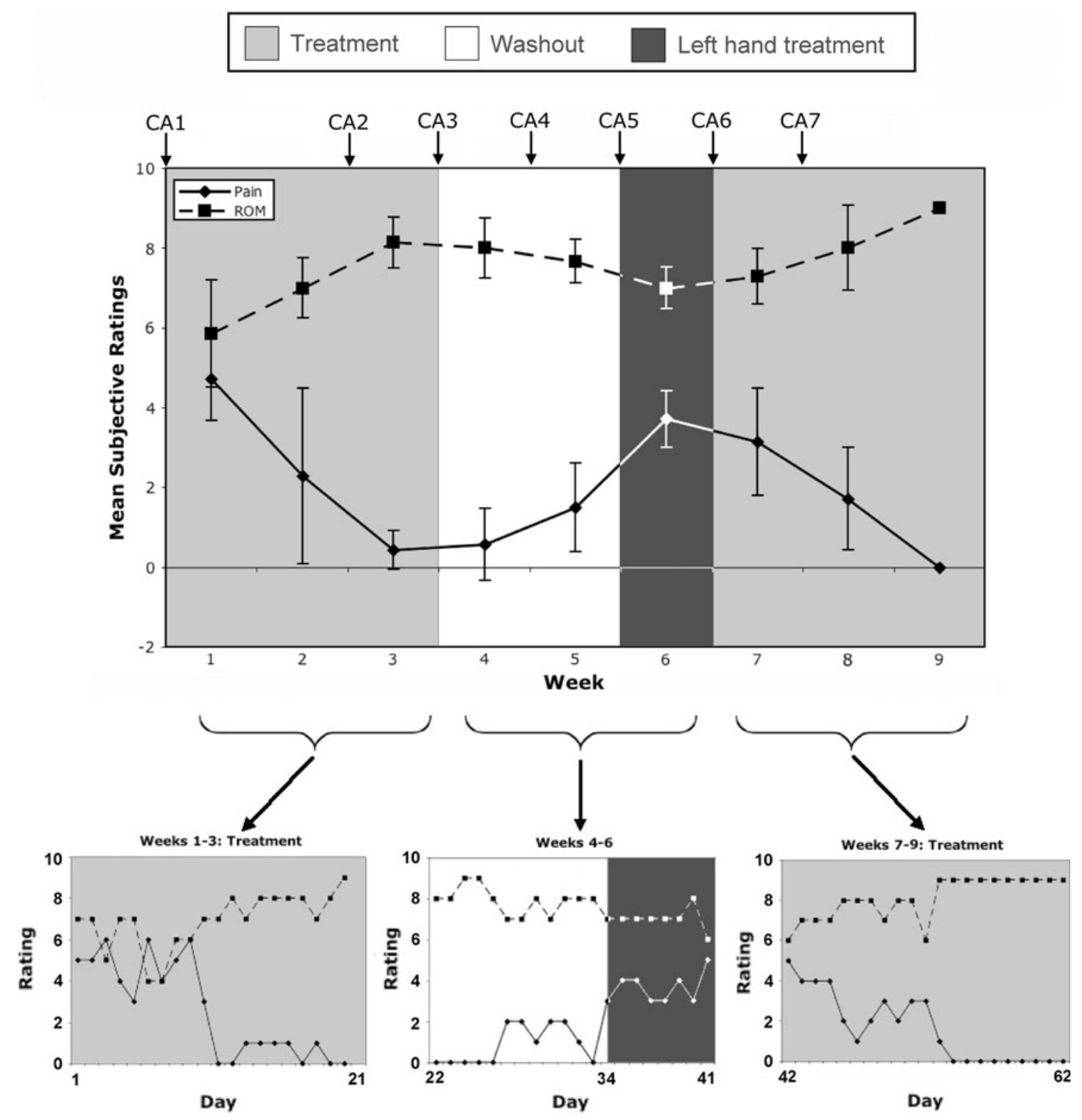

Fig. 2 Ratings of pain and range of movement (ROM) made by SM on each day for the first 9 weeks. The upper figure shows the ratings averaged over each week, with error bars indicating the $95 \%$ confidence interval around the mean. Daily ratings are detailed in the lower figures. Timing of the first seven clinical assessments are indicated

For prism adaptation SM wore welding goggles that were fitted with 25-diopter (approximately $17^{\circ}$ ) leftwardshifting Fresnel lenses. She made 50 alternate pointing movements to targets located at arm's length and shoulder height at approximately $10^{\circ}$ to the left and right of her midsagittal plane. She pointed as fast as possible with the index finger of her right hand, returning her hand to her torso in between each pointing movement. The first adaptation session was performed under experimenter guidance immediately after the baseline clinical assessment (CA1) to ensure $\mathrm{SM}$ understood the procedure. Comparison of errors for ten
(CA1-CA7). Ratings were made immediately prior to daily treatment sessions on two 11-point numerical rating scales (NRS) ranging from 0 ('no pain at all'/'no range of movement at all') to 10 ('pain as bad as it could be'/'full range of movement')

open-loop pointing movements performed immediately before and prism adaptation confirmed a significant $7.25^{\circ}$ rightward visuomotor after-effect $[t(9)=9.3, P<0.001]$. SM continued daily prism adaptation at home at approximately the same time each evening.

\section{Results}

The outcomes of the formal clinical assessments are outlined in Table 1, and SM's subjective ratings for pain and 


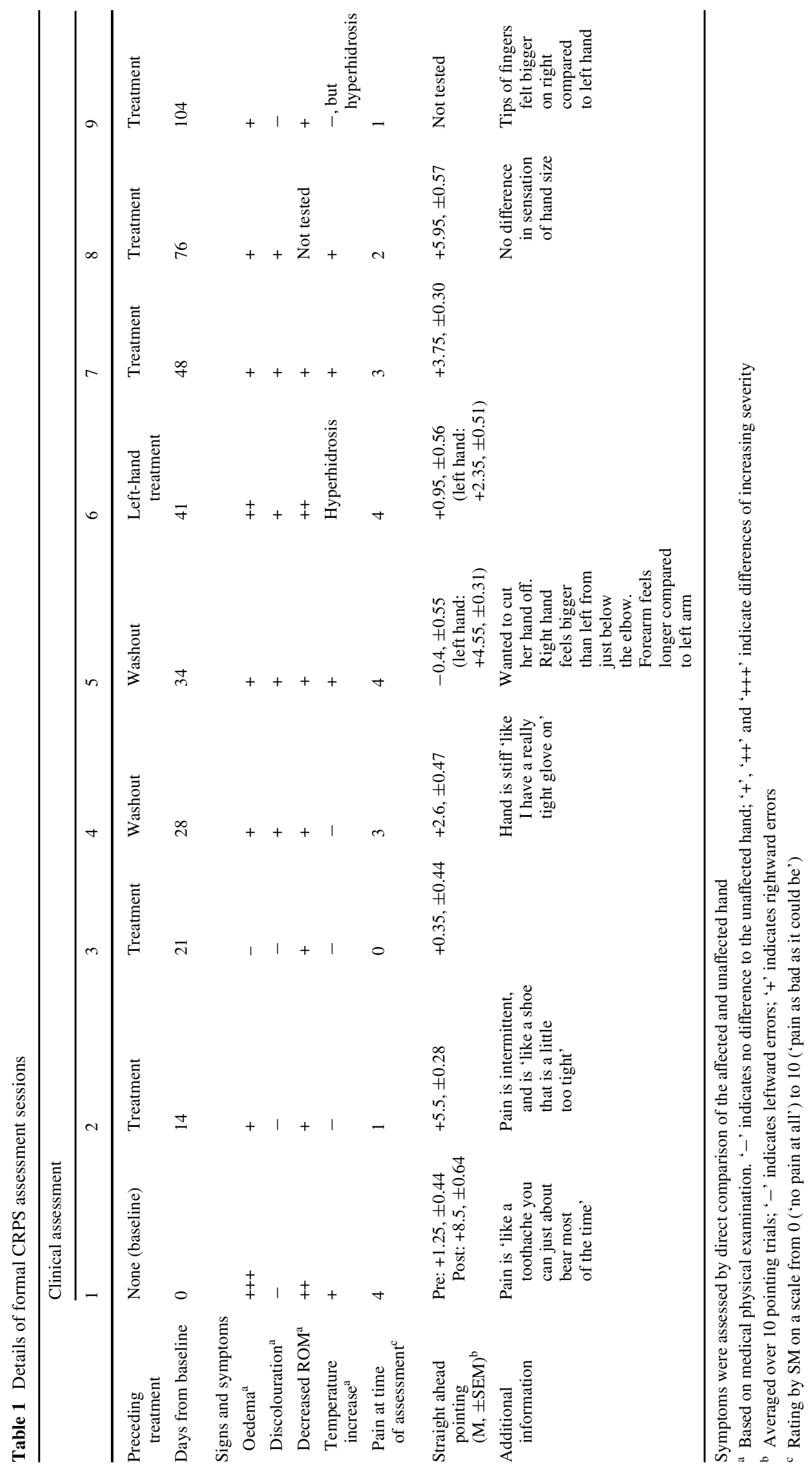


ROM are shown in Fig. 2. SM first underwent 3 weeks of adaptation to leftward-shifting prisms using her right hand. Clinical assessments performed at the end of weeks 2 and 3 (CA2 and CA3) revealed a progressive decrease in pain, swelling and temperature difference, as well as an increase in range of movement compared to the baseline clinical assessment (CA1). Within 9 days of the start of treatment the patient was pain free, requiring no pain relief medication. For the remainder of this treatment period pain remained absent $(\mathrm{NRS}=0)$, or was experienced only as minor pain ('like a shoe that is a little too tight', NRS $=1$ ), lasting for a few minutes and no longer than an hour. 95\% confidence intervals (CIs) constructed around the mean ratings for each week revealed that pain throughout the third week $(\mathrm{CI}=[-0.7,0.9])$ was significantly less than throughout the first week $(\mathrm{CI}=[3.7,5.7])$, and was also no different from zero (see Fig. 2). Similarly, there was a significant increase in $\mathrm{ROM}$ between the first $(\mathrm{CI}=[4.5,7.2])$ and third $(\mathrm{CI}=[7.5,8.8])$ week of treatment, although at the end of the third week SM was still not able to completely close her hand outside mirror therapy (NRS $=9$ ).

To determine whether the reduction in SM's pain and other symptoms was contingent upon continued treatment, prism adaptation was discontinued after the end of the third week ('washout'). The average pain rating over the first week of washout (week 4) was not significantly different from zero $(\mathrm{CI}=[-0.3,1.5])$ and SM remained pain free for 5 days (see Fig. 2). On the sixth day, pain returned. This was at first intermittent, but over the following days pain continued to increase in frequency and severity until on day 13 of the washout period SM once again had the need for medication for pain relief. Over the last 6 days of the washout period (week 5) pain was once again significantly greater than zero $(\mathrm{CI}=[0.4,2.6])$, but still significantly smaller than in week $1(\mathrm{CI}=[3.7,5.9])$. The increase in pain during washout was mirrored by a decrease in ROM (week 5: CI $=[7.1,8.2]$ ), which returned to week 1 levels $(\mathrm{CI}=[4.5,7.2])$. A gradual return of symptoms was also noted in clinical assessments 4 and 5, with a return of swelling, warmth and discolouration compared to the unaffected hand. Interestingly, the patient reported that in the final 2 days of the washout period she experienced occasional sharp jabbing pains in the palm of her left hand, which resembled the pain that she had first felt in her right hand at the onset of CRPS.

By the thirteenth day of the washout period symptoms had returned to a level that was clinically significant and caused SM some discomfort. We therefore decided to recommence treatment. To determine if prism adaptation could reduce symptoms if performed with the unaffected hand, SM volunteered to undertake daily adaptation sessions using her left hand ('left-hand treatment'). The procedure was otherwise identical to that described above.
Symptoms continued to worsen further to the deterioration observed during the washout period, with the $95 \%$ CIs for week 6 showing that average pain $(\mathrm{CI}=[3.0,4.4])$ and $\mathrm{ROM}(\mathrm{CI}=[6.5,7.5])$ were no different to the week 1 ratings (Fig. 2). Clinical assessment performed at the end of one week of left-hand treatment (CA6) revealed a stiff, sweaty and swollen hand. SM reported loss of function to pre-treatment levels, with greatly diminished power and pain that was not relieved by medication. This frustrated her a great deal and she spontaneously reported feeling like she wanted to cut her hand off. When SM closed her eyes her right arm from the elbow to the fingertips felt larger and the forearm longer compared to the left.

As there was no evidence for symptom relief from adaptation using the unaffected hand, we resumed adaptation with the affected hand to determine if the previous improvements would be recovered (weeks 7-9). Clinical assessment after week 7 (CA7) showed that swelling and temperature difference had once again decreased. In week 8 pain ratings were once again significantly less than in week $1(\mathrm{CIs}=[0.4,2.9]$ and $[3.7,5.7]$ respectively $)$, and pain reduced to zero after 13 days. There was a similar increase in ROM to NRS $=9$ by week 9 , significantly larger than in week 1 . Pain remained completely absent or mild and infrequent over a further 2 months of treatment (CA8 and CA9), with one notable exception: SM missed prism treatment for 2 days while on holiday and this was followed by a sharp rise in pain 2 days later (NRS $=5$ ) and a decrease in ROM (NRS $=7$ ), even though she had recommenced prism treatment. The pain subsided after 4 days. Sensory disturbance also decreased in the second treatment period; in the final two clinical assessments (CA8 and CA9) there was little or no difference between the felt size of the right hand relative to the left.

SM is continuing daily adaptation sessions, with a view to gradually decrease the frequency of treatments in the future if pain control is sustained. In both treatment periods the improvement in symptoms were accompanied by improvement in range of motion and function in everyday activities. She has recovered the ability to perform tasks requiring fine motor control or accurately applied pressure such as using a needle and thread, picking up a cup by its handle, bending wire with pliers or ironing a garment.

Although pain free for several weeks, she is still not able to fully close her hand, except when using a mirror; and when she closes her hand in the mirror, she is still unable to unclench the closed fist when the mirror is removed. When asked why she cannot open her hand, she seems bemused and can only respond that 'I don't know how'. She is unable to pick up a teapot or carry heavy bags. Thus, there is a continuing impairment of motor function that is not simply a limitation due to pain. 


\section{Discussion}

We find it interesting that in this particular patient there is a history of both phantom digit pain and CRPS. This may suggest that these syndromes are related, and that some individuals may be more susceptible to their development. CRPS is three times more common in women than in men and the highest incidence is in women who, like SM, are in middle age (de Mos et al. 2007). It is also possible that the traumatic concussion may have increased her vulnerability to development of the syndrome, as this 'commotion of the brain' (commotio cerebri; McCrory and Berkovic 2001) can trigger neuroplasticity and cortical reorganisation (Levin 2003; Anderson et al. 2006). As is typical of CRPS, her pain was greatly disproportional to the severity of the injury and began after the pain due to fracture had begun to subside. It is noteworthy that she experienced symptoms of body representation disturbance while the limb was immobilized before the pain of CRPS began. Her symptoms of CRPS included some that suggested a disturbance of body representation: distorted sense of limb size, lack of knowledge of limb location, and hostility towards the limb.

In two treatment periods, pain, stiffness and other CRPS signs and symptoms decreased within 2 weeks ( $\sim 10$ days), replicating the results of Sumitani et al. (2007). In addition, we found that the benefits of prism adaptation were maintained with continued treatment, symptoms worsened when treatment was discontinued, and that adaptation to leftwardshifting prisms using the left hand was ineffective.

It has recently been suggested that CRPS arises from incongruence between the true consequences of motor commands and the anticipated consequences-or efference copy-of those commands to the cortex (McCabe et al. 2003). With reference to pain from repetitive strain injury and phantom limb pain, Harris (1999) proposed that a distorted cortical representation of a limb can result in discrepancies between motor intention, proprioception and vision. This leads to the affective sensation of pain in a similar way to the development of nausea with conflicting visual and vestibular signals. In CRPS, these discrepancies are postulated to initiate protective and defensive sympathetic mechanisms through the autonomic nervous system (McCabe and Blake 2008). Several observations suggest that patients with CRPS have pathologically reorganised neural representations of peripersonal space that normalise with recovery. The sensory-motor discordance that is induced by prism lenses may provide an error signal that initiates the normalisation of body representation, reducing that component of pain that is driven by incongruence in motor intention, proprioception and vision.

Both our observations of SM and the results of Sumitani et al. (2007) suggest that this realignment requires at least several days of treatment. Sensory feedback from the affected hand during prism adaptation seems essential for therapeutic benefit, as SM's symptoms were not improved by adaptation with the left hand but continued to escalate to pre-treatment levels. It would be interesting to test whether mirror therapy and prism adaptation could be combined by asking patients to point to targets with their unaffected limb while viewing the reflected image of the unaffected limb through prismatic glasses. If it is possible to induce sensory-motor realignment in the affected limb using this technique, then prism adaptation may also hold therapeutic potential for relieving pain in phantom or hemiplegic limbs (that is, patients who would be unable to perform prism adaptation with their affected limb).

When treatment was discontinued pain returned to the affected hand and also began to emerge in the left (unaffected) hand. Emergence of CRPS symptoms in equivalent contralateral locations, or 'mirror-image' spread, has been reported and is a further argument against purely local inflammatory causes (Maleki et al. 2000). However, SM's history of amputation of the first and second digits of the left hand also leads us to speculate that as the prisminduced realignment faded there may have been a reawakening of phantom pain caused by the re-detection of the sensory-motor mismatch caused by the missing digits.

Finally, we note that although prism adaptation resulted in substantial relief of symptoms and return of function, it was not completely curative. At the final assessment there remained some swelling in the knuckles of the first and second finger, sweatiness of the palm and sensory disturbance. All of these symptoms were very mild and detected only on close comparison to the left hand. Pin pricks were experienced as blunt, nonpainful brushing of the skin. The residual restriction of movement was, however, more apparent. Alleviation of pain with prism adaptation, accompanied by mirror treatment that may have prevented atrophy and motor deterioration by enabling her full hand movements periodically, did achieve a sustained improvement in range of motion and functionality of the hand. However, SM is still not able to fully close her fingers, and it is therefore clear that there is a deficit in somatomotor function in this syndrome that is independent from pain. Noting that SM's symptoms of distorted body image began before her pain, we may speculate that the disturbance of central somatomotor circuits is the primary cause of the syndrome and the pain is secondary to it. This hypothesis, based on our observational case study, requires more systematic testing (for example, through retrospectively surveying the early symptoms of patients with CRPS). It remains to be seen whether continuing prism adaptation can ultimately restore normal function in limbs affected by CRPS. 


\section{Concluding remarks}

We report several observations that may offer insight into the pathophysiology of this enigmatic and vexing disease, and guidance in developing a therapeutic strategy.

1. In this patient, symptoms of body image disturbance preceded the development of other CRPS symptoms. We may speculate that, in some patients, CRPS is primarily a disturbance of body representation, and that pain is a consequence, not the cause, of the derangement of body representation. This may be a fruitful area for future research identifying subtypes of the disorder, and it is possible that such patients may be more likely to benefit from mirror therapy and prism adaptation treatment.

2. Physiological deconditioning due to disuse, including bone and muscle loss, has been well documented (Bortz 1982; Greenleaf 1984), and previous authors have proposed immobilisation as a direct cause of CRPS symptoms other than pain (Galer and Harden 2001). In our patient the symptoms of impaired body representation began while the limb was immobilised. We may speculate further that it is not pain, but rather immobilisation of the limb after trauma (whether due to splinting or limitations of movement secondary to pain) that precipitates a reorganisation of somatomotor circuits in susceptible individuals, leading to the disturbance in body representation.

3. Consistent with this account, although relief of pain after a course of prism adaptation improved range of motion that had been limited by pain, and restored much functionality to the limb, it did not restore the full repertoire of hand postures that could be produced voluntarily. Indeed, after closing her hand in the mirror, she was unable to unclench her fist outside the mirror, reporting that 'I don't know how'. It is clear then that the limitation of voluntary movement is neither due to pain, nor to weakness (since unclenching the fist would only require the ability to relax it). Rather, it appears that a derangement of body representation has resulted in the loss of certain postures from the repertoire of hand movements that can be produced by voluntary activation of somatomotor circuits. As is the case in patients with ideomotor apraxia, SM had better range of movement when using objects than when performing actions to verbal command. Unlike apraxia, however, SM did not produce an incorrect sequence of movements but, rather, experience paralysis. We can speculate that the mechanism of paralysis in this case might share similarities with conversion disorder.

4. The ability to execute movements in the mirror suggests that the capability of the unaffected hemisphere to generate these postures can be transferred to the affected hemisphere if the affected motor system is provided with visual information that can replace, bypass or dominate the disturbance of kinesthesis.

5. Although our observations do not yet offer a basis for optimism that prism adaptation may be curative, they do show that the treatment can provide sustained relief of pain, reduce disability and improve quality of life.

6. When SM underwent 1 week of adaptation using her unaffected hand, symptoms did not improve but returned to baseline. That is, the benefits of prism adaptation are contingent on adapting with the affected limb, suggesting that its benefits derive from perturbation of the dysfunctional somatomotor representation of that limb (compared to, for example, the general spatial representation of the body within extra-personal space).

7. In conjunction with mirror therapy to maintain the potential for full active range of motion, prism adaptation has the potential to prevent progression to a withered, useless limb, which might otherwise be the fate of patients with this disorder.

Acknowledgments This work was supported by the British Federation for Women Graduates (JHB) and the Wellcome Trust (RDR). We thank SM for her helpful insights and participation, and Betsy Rafal and Peter Maddison for their valuable suggestions.

\section{References}

AbuRahma AF, Robinson PA, Powell M, Bastug D, Boland JP (1994) Sympathectomy for reflex sympathetic dystrophy: factors affecting outcome. Ann Vasc Surg 8:372-379

Alexander GM, van Rijn MA, van Hilten JJ, Perreault MJ, Schwartzman RJ (2005) Changes in cerebrospinal fluid levels of proinflammatory cytokines in CRPS. Pain 116:213-219

Anderson T, Heitger M, Macleod AD (2006) Concussion and mild head injury. Pract Neurol 6:342-357

Berger P (1999) The role of the physiotherapist in the treatment of complex peripheral pain syndromes. Pain Rev 6:211-232

Bortz WM 2nd (1982) Disuse and aging. JAMA 248:1203-1208

Bruehl S, Carlson CR (1992) Predisposing psychological factors in the development of reflex sympathetic dystrophy. A review of the empirical evidence. Clin J Pain 8:287-299

Choi YS, Lee MG, Lee HM, Lee CJ, Jo JY, Jeon SY, Lee SC, Kim YC (2008) Epidemiology of complex regional pain syndrome: a retrospective chart review of 150 Korean patients. J Korean Med Sci 23:772-775

de Mos M, de Bruijn AG, Huygen FJ, Dieleman JP, Stricker BH, Sturkenboom MC (2007) The incidence of complex regional pain syndrome: a population-based study. Pain 129:12-20

Dijkerman HC, Webeling M, ter Wal JM, Groet E, van Zandvoort MJE (2004) A long-lasting improvement of somatosensory function after prism adaptation, a case study. Neuropsychologia 42:1697-1702

Doury P (1988) Algodystrophy. Reflex sympathetic dystrophy syndrome. Clin Rheumatol 7:173-180

Evans JA (1946) Reflex sympathetic dystrophy. Surg Clin N Am 26:780-790 
Forderreuther S, Sailer U, Straube A (2004) Impaired self-perception of the hand in complex regional pain syndrome (CRPS). Pain 110:756-761

Galer BS, Harden RN (2001) Motor abnormalities in CRPS: a neglected by key component. In: Harden RN, Baron R, Jänig W (eds) Complex regional pain syndrome. Progress in pain research and management, vol 22. IASP Press, Seattle, pp 135-140

Gordon N (1996) Reflex sympathetic dystrophy. Brain Dev 18:257262

Greenleaf JE (1984) Physiological responses to prolonged bed rest and fluid immersion in humans. J Appl Physiol 57:619-633

Huygen FJ, De Bruijn AG, De Bruin MT, Groeneweg JG, Klein J, Zijlstra FJ (2002) Evidence for local inflammation in complex regional pain syndrome type 1. Mediators Inflamm 11:47-51

Lam KK, Lee TW, Chen PP (1996) Complex regional pain syndrome type I. Hong Kong Med J 2:414-418

Levin HS (2003) Neuroplasticity following non-penetrating traumatic brain injury. Brain Inj 17:665-674

Lewis JS, Kersten P, McCabe CS, McPherson KM, Blake DR (2007) Body perception disturbance: a contribution to pain in complex regional pain syndrome (CRPS). Pain 133:111-119

Maihofner C, Handwerker HO, Neundorfer B, Birklein F (2003) Patterns of cortical reorganization in complex regional pain syndrome. Neurology 61:1707-1715

Maihofner C, Handwerker HO, Neundorfer B, Birklein F (2004) Cortical reorganization during recovery from complex regional pain syndrome. Neurology 63:693-701

Maleki J, LeBel AA, Bennett GJ, Schwartzman RJ (2000) Patterns of spread in complex regional pain syndrome, type I (reflex sympathetic dystrophy). Pain 88:259-266

Manning DC (2000) Reflex sympathetic dystrophy, sympathetically maintained pain, and complex regional pain syndrome: diagnoses of inclusion, exclusion, or confusion? J Hand Ther 13:260-268

Maravita A, McNeil J, Malhotra P, Greenwood R, Husain M, Driver J (2003) Prism adaptation can improve contralesional tactile perception in neglect. Neurology 60:1829-1831

McCabe CS, Blake DR (2008) An embarrassment of pain perceptions? Towards an understanding of and explanation for the clinical presentation of CRPS type 1. Rheumatology (Oxford) 47:1612-1616

McCabe CS, Haigh RC, Halligan PW, Blake DR (2003a) Referred sensations in patients with complex regional pain syndrome type 1 . Rheumatology (Oxford) 42:1067-1073

McCabe CS, Haigh RC, Ring EF, Halligan PW, Wall PD, Blake DR (2003b) A controlled pilot study of the utility of mirror visual feedback in the treatment of complex regional pain syndrome (type 1). Rheumatology (Oxford) 42:97-101

McCrory PR, Berkovic SF (2001) Concussion: the history of clinical and pathophysiological concepts and misconceptions. Neurology 57:2283-2289

McIntosh RD, Rossetti Y, Milner AD (2002) Prism adaptation improves chronic visual and haptic neglect: a single case study. Cortex 38:309-320
Mitchell SW, Morehouse GR, Keen WW (2007) Gunshot wounds and other injuries of nerves. 1864. Clin Orthop Relat Res 458:35-39

Moseley GL (2005) Distorted body image in complex regional pain syndrome. Neurology 65:773

Moseley GL, Parsons TJ, Spence C (2008) Visual distortion of a limb modulates the pain and swelling evoked by movement. Curr Biol 18:R1047-R1048

Oaklander AL (2008) RSD/CRPS: the end of the beginning. Pain 139:239-240

Pisella L, Rode G, Farnè A, Boisson D, Rossetti Y (2002) Dissociated long lasting improvements of straight-ahead pointing and line bisection tasks in two hemineglect patients. Neuropsychologia 40:327-334

Pleger B, Tegenthoff M, Ragert P, Forster AF, Dinse HR, Schwenkreis $P$, Nicolas V, Maier C (2005) Sensorimotor retuning in complex regional pain syndrome parallels pain reduction. Ann Neurol 57:425-429

Ramachandran VS, Rogers-Ramachandran D, Cobb S (1995) Touching the phantom limb. Nature 377:489-490

Rossetti Y, Rode G, Pisella L, Farnè A, Li L, Boisson D, Perenin M (1998) Prism adaptation to a rightward optical deviation rehabilitates left hemispatial neglect. Nature 395:166-169

Sarri M, Greenwood R, Kalra L, Papps B, Husain M, Driver J (2008) Prism adaptation aftereffects in stroke patients with spatial neglect: pathological effects on subjective straight ahead but not visual open-loop pointing. Neuropsychologia 46:1069-1080

Schasfoort FC, Bussmann JB, Stam HJ (2004) Impairments and activity limitations in subjects with chronic upper-limb complex regional pain syndrome type I. Arch Phys Med Rehabil 85:557-566

Stanton-Hicks M, Janig W, Hassenbusch S, Haddox JD, Boas R, Wilson P (1995) Reflex sympathetic dystrophy: changing concepts and taxonomy. Pain 63:127-133

Sudeck P (1900) Ueber die acute entzundliche knochenatrophie. Arch Klin Chir 62:147

Sumitani M, Rossetti Y, Shibata M, Matsuda Y, Sakaue G, Inoue T, Mashimo T, Miyauchi S (2007) Prism adaptation to optical deviation alleviates pathologic pain. Neurology 68:128-133

Tichelaar YIGV, Geertzen JHB, Keizer D, van Wilgen CP (2007) Mirror box therapy added to cognitive behavioural therapy in three chronic complex regional pain syndrome type I patients: a pilot study. Int J Rehabil Res 30:181-188

Torebjork E, Wahren L, Wallin G, Hallin R, Koltzenburg M (1995) Noradrenaline-evoked pain in neuralgia. Pain 63:11-20

van der Laan L, Veldman PH, Goris RJ (1998) Severe complications of reflex sympathetic dystrophy: infection, ulcers, chronic edema, dystonia, and myoclonus. Arch Phys Med Rehabil 79:424-429

Veldman PH, Reynen HM, Arntz IE, Goris RJ (1993) Signs and symptoms of reflex sympathetic dystrophy: prospective study of 829 patients. Lancet 342:1012-1016

Wasner G, Schattschneider J, Binder A, Baron R (2003) Complex regional pain syndrome-diagnostic, mechanisms, CNS involvement and therapy. Spinal Cord 41:61-75 